\title{
Sala de aula invertida no ensino superior de saúde: uma revisão sistemática
}

\author{
Flipped classroom in higher health education: a systematic review \\ El aula invertida en la educación superior en salud: una revisión sistemática
}

\section{Resumo}

O presente estudo tem como objetivo identificar as contribuições da sala de aula invertida no processo de ensinoaprendizagem de professores e alunos da área da saúde. Trata-se de uma revisão sistemática. A questão norteadora foi "Quais as contribuições da sala de aula invertida na formação em saúde?” em seguida, realizou-se a busca em quatro bases eletrônicas: a Literatura Latino-Americana e do Caribe em Ciências da Saúde (LILACS), a Medical Literature Analysis and Retrieval System Online (MEDLINE/PubMed), a Banco de Dados de Enfermagem (BDENF), e a Scientific Electronic Library Online (SciElo). Foram incluídos 77 estudos. A maioria das publicações estavam relacionadas ao nível de ensino da graduação $(n=57)$ seguido da pós graduação (especialização, residência, mestrado e doutorado) $(n=20)$. Com utilização de sala invertida no contexto da área médica $(n=55)$, de odontologia $(n=11)$, de enfermagem $(n=8)$, de fisioterapia $(n=1)$, de farmácia $(n=2)$. As principais contribuições com o ensino superior em saúde foram o aumento dos resultados de aprendizagem e da capacidade de compreensão dos assuntos, a preparação prévia para as aulas presenciais, a variabilidade de recursos tecnológicos e materiais, a possibilidade de realizar atividades didáticas interativas. Identificou-se no estudo, que a utilização da sala de aula invertida promove autonomia, estudo autodirigido e desenvolvimento de habilidades através da aprendizagem combinada. Faz-se necessário treinamentos voltados para os professores a fim de estimulá-los a utilizarem a estratégia de ensino e aprendizagem da sala de aula invertida.

Palavras-chave: Aprendizagem; Ensino; Ensino superior.

\begin{abstract}
This study aims to identify the contributions of the inverted classroom in the teaching-learning process of health care teachers and students. This is a systematic review. The guiding question was "What are the contributions of the inverted classroom in health education?" then, a search was carried out in four electronic databases: the Latin American and Caribbean Literature on Health Sciences (LILACS), the Medical Literature Analysis and Retrieval System Online (MEDLINE/PubMed), the Nursing Database (BDENF), and the Scientific Electronic Library Online (SciElo). 77 studies were included. Most publications were related to the undergraduate level of education $(n=57)$ followed by postgraduate education (specialization, residency, master's and doctorate) $(n=20)$. With the use of an inverted room in the context of the medical area $(n=55)$, dentistry $(n=11)$, nursing $(n=8)$, physiotherapy $(n=1)$, pharmacy $(n=2)$. The main contributions to higher education in health were the increase in learning outcomes and the ability to understand the subjects, prior preparation for classroom classes, the variability of technological and material resources, the possibility of carrying out interactive teaching activities. It was identified in the study that the use of the inverted classroom promotes autonomy, self-directed study and skills development through blended learning. Training aimed at teachers is necessary in order to encourage them to use the inverted classroom teaching and learning strategy.
\end{abstract}

Keywords: Learning; Teaching; University education.

\section{Resumen}

Este estudio tiene como objetivo identificar las contribuciones del aula invertida en el proceso de enseñanza-aprendizaje de docentes y estudiantes de salud. Ésta es una revisión sistemática. La pregunta orientadora fue "¿Cuáles son las 
contribuciones del aula invertida en la educación para la salud?" Luego, se realizó una búsqueda en cuatro bases de datos electrónicas: la Literatura Latinoamericana y del Caribe en Ciencias de la Salud (LILACS), el Sistema de Análisis y Recuperación de Literatura Médica en Línea (MEDLINE / PubMed), la Base de Datos de Enfermería (BDENF) y la Base de Datos Científica Electrónica. Biblioteca en línea (SciElo). Se incluyeron 77 estudios. La mayoría de las publicaciones se relacionaron con el nivel de educación de pregrado $(n=57)$ seguido de la educación de posgrado (especialización, residencia, maestría y doctorado $(\mathrm{n}=20)$. Con el uso de sala invertida en el contexto del área médica $(\mathrm{n}=55)$, odontología $(\mathrm{n}=11)$, enfermería $(\mathrm{n}=8)$, fisioterapia $(\mathrm{n}=1)$, farmacia $(\mathrm{n}=2)$. Los principales aportes a la educación superior en salud fueron el aumento de los resultados de aprendizaje y la capacidad de comprensión de las asignaturas, la preparación previa a las clases presenciales, la variabilidad de los recursos tecnológicos y materiales, la posibilidad de realizar actividades didácticas interactivas. En el estudio se identificó que el uso del aula invertida promueve la autonomía, el estudio autodirigido y el desarrollo de habilidades a través del aprendizaje mixto. La formación dirigida a los profesores es necesaria para animarlos a utilizar la estrategia de enseñanza y aprendizaje del aula invertida.

Palabras clave: Aprendizaje; Enseñando; Enseñanza superior.

\section{Introdução}

No início de 2020 a Organização Mundial da Saúde (OMS) declarou estado de emergência pública devido à doença causada pelo coronavírus, a COVID-19. Durante o ano, a situação foi agravada e tomou grandes proporções epidemiológicas, o que causou impacto mundial, sendo necessário a criação de políticas públicas de prevenção e precaução como o distanciamento e isolamento social, a fim de minimizar o impacto da pandemia. Tais medidas trouxeram várias mudanças e desafios no setor econômico, social e educacional (Organização Pan-Americana da Saúde/OMS 2020).

Frente a situação, o ensino superior impossibilitado para a execução de aulas presenciais redirecionou suas estratégias e buscou soluções para dar continuidade às atividades acadêmicas, sendo aulas de forma remota emergencial uma estratégia proposta. Hodges e colaboradores (2020) explicam que o Ensino Remoto Emergencial (ERE) é caracterizado por uma mudança temporária da forma de ensino, para um modo alternativo devido a circunstâncias de crise. Os mesmos consideram que essa abordagem deve envolver o uso de soluções de ensino totalmente remotas para o processo educacional ou através da forma híbrida.

Assim, a educação remota emergencial pode ser apresentada de forma similar à educação presencial, como a transmissão em horários específicos das aulas dos professores, nos formatos de lives. Tal transmissão permitiria a colaboração e participação de todos de forma simultânea, mas pode envolver a gravação das atividades para serem acompanhadas por alunos sem condições de assistir aos materiais naquele momento, através da implementação de ferramentas assíncronas (que funcionam de forma não instantânea, como fóruns de discussão) e disponibilização de materiais no formato online (Arruda, 2020).

Nessa perspectiva, a Sala de Aula Invertida, consiste em uma estratégia de ensino e aprendizagem, no qual utiliza-se de dois momentos de atividades. No primeiro momento, o aluno utiliza o formato online para estudar aspectos teóricos em casa, no segundo momento o espaço da sala de aula é utilizado para discussão em equipe e resolução de problemas. De modo geral, o aluno tem o contato prévio com o conteúdo, que serve como base para as discussões em sala. Sendo assim, a sala de aula invertida entra como uma estratégia de grande potencial para melhorar o ambiente de aprendizagem, proporcionar maior interação, autonomia para o aluno e organização do tempo do aluno e do professor (Bacich et al., 2015; Schneider et al., 2013).

Em contraste, o modelo e os conceitos de ensino tradicional utilizado nos cursos transmitem conhecimento através de aulas expositivas, por exemplo, ou em outros formatos de ensino presencial e são seguidos pela auto instrução dos alunos para assimilar esse conhecimento. Em decorrência disso, a finalidade da sala de aula invertida é a mudança da aprendizagem passiva para a aprendizagem acelerada, a fim de promover a aprendizagem em níveis cognitivamente exigentes, como análise, síntese e avaliação do conteúdo abordado (Schneider et al., 2013; Halasa et al., 2020).

Diante do exposto, o objetivo deste estudo foi identificar quais as contribuições da sala de aula invertida no processo de ensino-aprendizagem de professores e alunos da área da saúde, em especial nas áreas da enfermagem, medicina, odontologia 
e fisioterapia.

\section{Metodologia}

Trata-se de uma revisão sistemática, tipo de revisão que fornece informações sobre as evidências relacionadas a um estudo primário para responder a uma questão norteadora, baseada na aplicação de métodos de busca, apreciação crítica e síntese dos dados obtidos (Brasil, 2012). A pergunta da pesquisa foi formulada de acordo com o acrônimo PICO (Santos, 2007), onde a população foi composta pelos estudantes e professores da área de saúde, a intervenção foi a utilização da sala de aula invertida, o controle foi o ensino tradicional e o desfecho foi a contribuição da sala de aula invertida no processo de aprendizagem. Diante da utilização do acrônimo, foi elaborada a questão norteadora "Quais as contribuições da sala de aula invertida na formação em saúde?"

Realizou-se a busca em quatro bases eletrônicas: a Literatura Latino-Americana e do Caribe em Ciências da Saúde (LILACS), a Medical Literature Analysis and Retrieval System Online (MEDLINE/PubMed), a Banco de Dados de Enfermagem (BDENF), e a Scientific Electronic Library Online (SciElo). Utilizou-se os descritores selecionados a partir dos Descritores em Ciências da Saúde (Decs): "Saúde", "Enfermagem", "Medicina", “Odontologia" e "Fisioterapia" e o termo derivado "Sala de aula invertida". E no Medical SubjectHeadings (MeSH): "Health education", "Medical education”, "Nursing education”, "Dentistry" e "Physical therapists" e o termo derivado "flipped classroom". O estudo foi limitado às áreas da enfermagem, medicina, odontologia e fisioterapia em função dos próprios dados, visto que estas apresentaram uma maior concentração de estudos em relação à área da saúde no geral.

O processo de busca ocorreu entre o mês de julho a outubro de 2020. A busca foi realizada por pares e, posteriormente, individualmente para que possíveis diferenças fossem encontradas. Para garantir a combinação entre os termos, utilizou-se o operador booleano AND. Efetuou-se as combinações em português: (Sala de aula invertida) AND (Saúde); (Sala de aula invertida) $A N D$ (Enfermagem); (Sala de aula invertida) AND (Medicina); (Sala de aula invertida) AND (Odontologia); (Sala de aula invertida) AND (fisioterapia); e em inglês: (Flipped classroom) AND (Health education [mesh terms]); (Flipped classroom) AND (Nursing education [mesh terms]); (Flipped classroom) AND (Medical education [mesh terms]); (Flipped classroom) AND (Dentistry [mesh terms]); (Flipped classroom) AND (Physical therapits [mesh terms]).

Como critérios de elegibilidade foram adotados: artigos completos publicados em revistas científicas, no período de 2015 a 2020, nos idiomas Português, Inglês ou Espanhol. Foram definidos como critérios de exclusão: (a) artigos do tipo editorial, revisão de literatura, artigos de reflexão, teses e dissertações; (b) artigos cujo conteúdo não estava diretamente relacionado à questão norteadora desta pesquisa.

O processo de seleção dos artigos seguiu as orientações da PRISMA (Preferred Reporting Items for Systematic Reviews and Meta-Analyses), para o processo de identificação, seleção, elegibilidade e inclusão dos estudos (Page et al., 2021).

Para a organização dos dados extraídos foi utilizada uma planilha padrão do Google Sheets® e analisados a partir dos seguintes elementos: identificação do artigo (autores, ano, país), Título do artigo, base de dados em que o artigo foi encontrado, objetivo da pesquisa, desenho e métodos, amostra, principais resultados e conclusões encontrados, tema central, modelo de ensino híbrido adotado (sala de aula invertida - técnicas utilizadas) e o nível de ensino em que o modelo de sala de aula invertida foi aplicado.

Para classificar o nível de evidência dos artigos, foi utilizado o sistema GRADE (Grading of Recommendations Assessment, Development and Evaluaton) segundo o qual, a qualidade da evidência é classificada em quatro níveis: alto, moderado, baixo, muito baixo. Esses níveis representam a confiança na estimativa dos efeitos apresentados no estudo. Com o sistema GRADE, a avaliação da qualidade da evidência é realizada para cada desfecho analisado. A classificação inicial da qualidade da evidência é definida a partir do delineamento dos estudos, por exemplo, se o estudo for um ensaio clínico 
randomizado o nível de evidência é alto, já quando apenas estudos observacionais são incluídos, a qualidade da evidência se inicia como baixa.

A pesquisa resultou na identificação inicial de 901 artigos. Em seguida, 87 artigos duplicados foram excluídos. Posteriormente, com a utilização do filtro foram excluídos 231 artigos. Por meio da leitura dos títulos e resumos, permaneceram 195 artigos elegíveis. Dos 195 artigos elegíveis, foi realizada a leitura minuciosa e integral de 192 artigos, dos quais 115 foram excluídos por não responderem à questão da pesquisa. Ao final, foram incluídos 77 estudos que respondiam à questão norteadora desta investigação, exposto no diagrama de fluxo que detalha este processo ilustrado na Figura 1.

Dos 77 estudos, 72 artigos (93,5\%) estavam presentes na MEDLINE/Pubmed e 5 artigos (6,5\%) encontravam-se na LILACS. A maior parte dos artigos foram publicados no ano de 2017 (n=22, 28,57\% dos artigos), 2020 ( $\mathrm{n}=16,20,80 \%$ dos artigos), seguida do ano $2018(\mathrm{n}=15,19,48 \%$ dos artigos), $2016(\mathrm{n}=11,14,28 \%$ dos artigos), 2019 ( $\mathrm{n}=11,14,28 \%$ dos artigos) e 2015 ( $n=2,2,59 \%$ dos artigos). As características gerais dos artigos incluídos neste estudo encontram-se detalhadas na Tabela 1. Das publicações incluídas, 22 estudos eram qualitativos (28,57\%), 16 quantitativos (20,77\%) e 39 estudos mistos (50,64\%).

Figura 1. Fluxograma do processo de seleção dos estudos segundo a recomendação PRISMA.

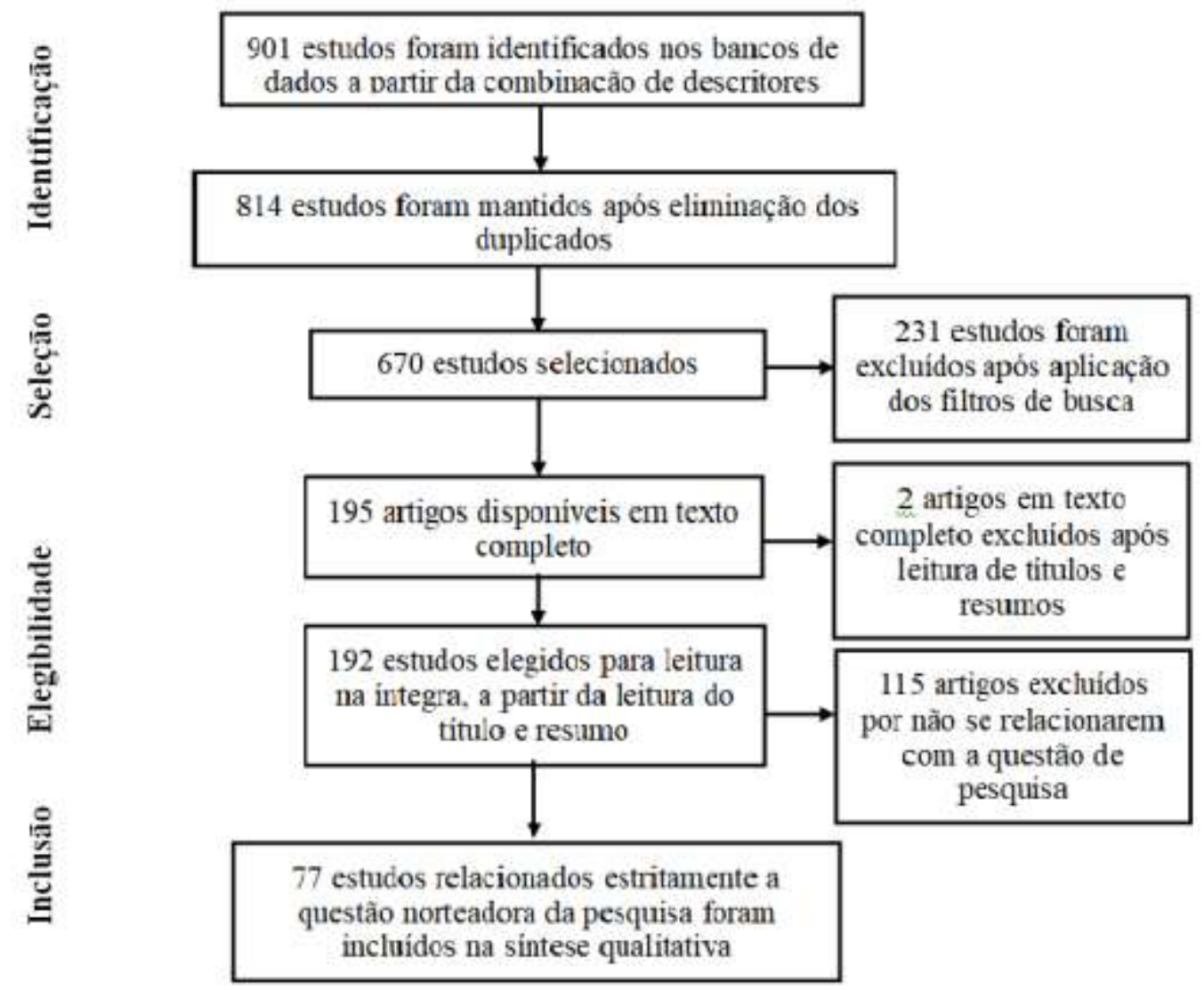

Fonte: Adaptado de Moher et al. (2009).

\section{Resultados}

Foram encontradas experiências com a utilização de sala invertida no contexto da área médica (n=55), de odontologia $(n=11)$, de enfermagem $(n=8)$, de fisioterapia $(n=1)$, de farmácia $(n=2)$. A maioria das publicações estavam relacionadas ao nível de ensino da graduação $(\mathrm{n}=57)$, seguido da pós graduação (especialização, residência, mestrado e doutorado) (n=20). As características gerais dos estudos selecionados encontram-se na Tabela 1. 
Tabela 1. Caracterização dos estudos incluídos na revisão sistemática sobre a sala de aula invertida. Recife - PE, 2020 (n=77).

\begin{tabular}{|c|c|}
\hline $\begin{array}{c}\text { Autor (Ano). } \\
\text { País. }\end{array}$ & Título do estudo \\
\hline $\begin{array}{l}\text { Oliveira et al., } \\
\text { 2018. Brasil }\end{array}$ & $\begin{array}{l}\text { Team-Based Learning como forma de aprendizagem } \\
\text { colaborativa e sala de aula invertida com centralidade nos } \\
\text { estudantes no processo ensino-aprendizagem }\end{array}$ \\
\hline $\begin{array}{l}\text { Melo Júnior et al., } \\
\text { 2018. Brasil }\end{array}$ & $\begin{array}{l}\text { Sala de aula invertida para o ensino do conteúdo abertura } \\
\text { coronária em Endodontia }\end{array}$ \\
\hline $\begin{array}{l}\text { Menegaz et al., } \\
\text { 2018. Brasil }\end{array}$ & $\begin{array}{l}\text { Flipped Classroom no ensino de gerenciamento em } \\
\text { enfermagem: relato de experiência }\end{array}$ \\
\hline $\begin{array}{l}\text { Andrés, } 2018 . \\
\text { Colômbia }\end{array}$ & $\begin{array}{l}\text { Impacto académico de una estrategia de salón invertido en } \\
\text { Anatomía }\end{array}$ \\
\hline $\begin{array}{l}\text { Domínguez et al., } \\
2015 . \\
\text { Colômbia }\end{array}$ & $\begin{array}{l}\text { Impacto de la estrategia de aula invertida en el ambiente de } \\
\text { aprendizaje en cirugía: una comparación con la clase magistral }\end{array}$ \\
\hline $\begin{array}{l}\text { Tang et al., } 2017 . \\
\text { EUA }\end{array}$ & $\begin{array}{l}\text { Comparison between flipped classroom and lecture-based } \\
\text { classroom in ophthalmology clerkship }\end{array}$ \\
\hline
\end{tabular}

Marco et al., 2017. Does the acceptance of hybrid learning affect learning França

Bas-Sarmient et al., 2017. Espanha

Jesurasa et al., 2017. Reino Unido

Katz et al., 2017. EUA

Rui et al., 2017.

China

Lucardie et al., 2017. Nova

Zelândia

Bonnes et al., 2017. EUA

Fleagle et al., 2018. EUA

Girgis \& Miller 2018. EUA

Martinelli et al., 2017. EUA

Riddell et al., 2017. EUA

Carrick et al., 2017. Reino Unido

Lin et al., 2017.China approaches in France?

Efficacy of empathy training in nursing students: A quasiexperimental study used in the preparation for postgraduate medical membership examinations?

Teaching procedural skills to medical students: A pilot procedural skills lab

Friend or Foe? Flipped Classroom for Undergraduate Electrocardiogram Learning: a Randomized Controlled Study

Flipping the classroom to teach Millennial residents medical leadership: a proof of concept

Flipping the Quality Improvement Classroom in Residency Education

Application of flipped classroom pedagogy to the human gross anatomy laboratory: Student preferences and learning outcomes

Implementation of a "Flipped Classroom" for Neurosurgery Resident Education

Results of a Flipped Classroom Teaching Approach in Anesthesiology Residents

Does the Flipped Classroom Improve Learning in Graduate Medical Education?

Randomized Controlled Study of a Remote Flipped Classroom Neuro-otology Curriculum

Facing the challenges in ophthalmology clerkship teaching: Is flipped classroom the answer?
What factors facilitate the engagement with flipped classrooms

\section{Desenho do estudo. Área de formação}

Relato de experiência. Estudantes de graduação em medicina $(n=38)$

Estudo prospectivo. Estudantes de graduação em odontologia $(n=14)$

Relato de experiência. Estudantes de graduação em enfermagem $(n=67)$

Estudo prospectivo comparativo. Estudantes de graduação em medicina $(n=71)$

Estudo de coorte. Estudantes de graduação em medicina $(n=207)$

Estudo prospectivo comparativo. Estudantes de graduação em medicina $(n=95)$
Moderado

Baixo

Alto

Alto

Alto

Estudo prospectivo observacional. Estudantes de graduação em medicina $(n=38)$

Estudo quase experimental. Estudantes de graduação em enfermagem $(n=49)$

Estudo exploratório. Estudante de pós-graduação em medicina $(n=14)$

Estudo de coorte transversal. Estudantes de graduação em medicina $(n=180)$

Ensaio clínico randomizado. Estudantes de graduação em medicina $(n=181)$

Estudo de coorte. Estudantes de pós-graduação em medicina

Estudo prospectivo. Estudantes de pós-graduação em medicina $(n=143)$

Estudo de coorte. Estudantes de pós-graduação em odontologia $(n=241)$

Estudo prospectivo. Estudantes de pós-graduação em medicina

$(n=12)$

Estudo prospectivo. Estudantes de pós-graduação em medicina $(n=182)$

Estudo randomizado. Estudantes de pós-graduação em medicina $(n=82)$

Estudo controlado randomizado. Estudantes de pós-graduação em medicina $(\mathrm{n}=274)$

Estudo prospectivo. Estudantes de graduação em medicina $(n=44)$
Alto

Alto

Alto

Alto

Alto

Alto

Moderado

Alto

Moderado

Moderado

Alto

Alto

Moderado 
Cheng et al., 2016. The "flipped classroom" approach: Stimulating positive China

Hsu et al., 2016.Taiwan

Lew, 2016. EUA

Sajid et al., 2016. Arabia saudita

Ferrer-Torregrosa

et al., 2016.

Inglaterra

Lochner et al.

2016. Itália

Tan et al., 2015

Nova Zelândia

El-Banna et al.,

2017. EUA

Green \& Schlairet 2017. EUA

Ihm et al., 2017

Coreia do sul

Nishigawa et al.,

2017. Japão

Bossaer et al., 2016. EUA

Ridell et al., 2016. Low Back Pain in the Emergency Medicine Department: A EUA

Flipped Classroom Module

Lossius et al., 2016. EUA

Boysen-Osborn et al., 2016. EUA

Mc Lean et al., 2016. Inglaterra

Gillispie, 2016. EUA

Gopalan \& klann, 2017. EUA

King et al., 2017. EUA

Phillips et al. 2017. EUA

learning attitudes and improving mastery of histology among medical students

An Investigation of the Outcomes of PGY Students' Cognition of and Persistent Behavior in Learning through the Intervention of the Flipped Classroom in Taiwan

Creating a contemporary clerkship curriculum: the flipped classroom model in emergency medicine

Can blended learning and the flipped classroom improve student learning and satisfaction in Saudi Arabia?

Distance learning ects and flipped classroom in the anatomy learning: comparative study of the use of augmented reality, video and notes

Combining traditional anatomy lectures with e-learning activities: how do students perceive their learning experience?

Acceptability of the flipped classroom approach for in-house teaching in emergency medicine

Flipping around the classroom: Accelerated Bachelor of Science in Nursing students' satisfaction and achievement

Moving toward heutagogical learning: Illuminating undergraduate nursing students' experiences in a flipped classroom

Flipped-learning course design and evaluation through student self-assessment in a predental science class

Comparison between flipped classroom and team-based learning in fixed prosthodontic education

Student Performance in a Pharmacotherapy Oncology Module Before and After Flipping the Classroom

An Interprofessional Small-Group Learning Experience in Early Childhood Caries With Fluoride Varnish Application

Flipping the Advanced Cardiac Life Support Classroom with Team-based Learning: Comparison of Cognitive Testing Performance for Medical Students at the University of California, Irvine, United States

Flipped classrooms and student learning: not just surface gains

Using the Flipped Classroom to Bridge the Gap to Generation Y

The effect of flipped teaching combined with modified teambased learning on student performance in physiology

Replacing Lectures with Small Groups: The Impact of Flipping the Residency Conference Day

Video-Based Teaching of Image-Guided Breast Interventions: Stereotactic Core Biopsy Using a Prone Table
Estudo randomizado. Estudantes de graduação em

Alto medicina $(n=24)$

Estudo prospectivo. Estudantes de pós-graduação

Moderado em medicina $(n=39)$

Estudo prospectivo. Estudantes de pós-graduação

Moderado em medicina $(n=46)$

Estudo analítico descritivo. Estudantes de graduação em medicina $(n=127)$

Estudo transversal quase experimental. Estudantes de graduação em medicina $(n=171)$

Estudo qualitativo. Estudantes de graduação em medicina $(n=36)$

Estudo qualitativo transversal. Estudantes de pósgraduação em medicina $(n=49)$

Estudo misto. Estudantes de graduação em enfermagem $(n=76)$

Estudo qualitativo. Estudantes de graduação em enfermagem $(n=14)$

Estudo misto. Estudantes de graduação em odontologia

Estudo prospectivo comparativo. Estudantes de graduação em odontologia $(n=41)$

Estudo prospectivo. Estudantes de graduação em farmácia $(\mathrm{n}=77)$

Estudo Misto. Estudantes de pós-graduação em medicina $(\mathrm{n}=42)$

Estudo Misto. Estudantes de graduação em medicina e odontologia $(n=210)$ e professores $(\mathrm{n}=17)$

Estudo prospectivo comparativo. Estudantes de graduação em medicina $(n=95)$

Estudo qualitativo. Estudantes de graduação em medicina $(n=54)$

Estudo prospectivo randomizado. Estudantes de graduação em medicina $(\mathrm{n}=70)$

Estudo prospectivo. Estudantes de graduação em enfermagem $(\mathrm{n}=178)$

Estudo transversal. Estudantes de pós-graduação em medicina $(n=18)$

Estudo misto. Estudantes de pós-graduação em medicina $(n=40)$
Alto

Alto

Alto

Moderado

Moderado

Moderado

Moderado

Moderado

Alto

Moderado

Moderado

Moderado

Moderado

Alto

Moderado

Alto

Moderado 
Fátima et al., 2017. Flipped classroom instructional approach

Paquistão in undergraduate medical education

Petit et al., 2017. What millennial medical students say about flipped learning

EUA

Kim et al., 2018.

Coréia

Seo et al., 2018

Coreia

Martinelli, 2018.

EUA

Xião et al., 2018

EUA

Gopalan et al.

2018. EUA

Wu et al., 2018.

Taiwan

Jung, 2018. Coreia Analysis of satisfaction and academic achievement of medical students in a flipped class

Beom et al., 2018. Flipped-classroom training in advanced

Coreia

cardiopulmonary life support

Lee \& Kim, 2018. Enhancement of student perceptions of

Coreia do Sul learner-centeredness and community of inquiry in flipped classrooms

Kohli et al., 2019. Dental education: Lecture versus flipped and spaced learning Malásia

Cho \& Kim, 2019. Outcomes and influential factors applying flipped learning

Coréia

Chu et al., 2018. The effects of the flipped classroom in teaching evidence based Taiwan

Roe et al., 2019.

Noruega

Salabe \&

Chowdary, 2019. Índia

He et al., 2019.

Reino Unido

Kühl et al., 2019.

Alemanha

Graham et al.,

2019.

EUA

Sohn et al., 2019.

Coreia implementation and evaluation of a flipped classroom teaching approach

Flipped classroom - An innovative teaching model to train undergraduate medical students in community medicine pharmaceutical marketing learning: A clustered randomized controlled study

Investigating the self-study phase of an inverted biochemistry classroom - collaborative dyadic learning makes the difference
Estudo misto. Estudantes de graduação em

Moderado medicina

$(\mathrm{n}=98)$

Estudo qualitativo. Estudantes de graduação em medicina $(n=104)$

Estudo misto. Estudantes de graduação em odontologia

Estudo exploratório. Estudantes de graduação em odontologia $(n=69)$

Estudo misto. Professores de anestesiologia $(n=244)$

Estudo qualitativo. Estudantes de graduação em odontologia

Estudo experimental. Professores de fisiologia $(\mathrm{n}=12)$

Estudo misto. Estudantes de graduação em medicina

$(\mathrm{n}=78)$

Estudo prospectivo. Estudantes de graduação em medicina $(\mathrm{n}=40)$

Estudo prospectivo. Comparativo Estudantes de graduação em medicina $(\mathrm{n}=108)$

Estudo prospectivo. Estudantes de graduação em medicina

$(\mathrm{n}=55)$

Estudo prospectivo de coorte.

Estudantes de graduação em odontologia $(n=60)$

Estudo prospectivo de coorte. Estudantes de graduação em enfermagem $(n=80)$

Estudo prospectivo. Estudantes de pós-graduação em enfermagem $(n=151)$

Estudo de coorte prospectivo. Estudantes de graduação em fisioterapia $(n=54)$

Estudo qualitativo. Estudantes de graduação em medicina

$(n=48)$

Estudo controlado randomizado. Estudantes de graduação em farmácia $(n=137)$

Estudo prospectivo. Estudantes de graduação em medicina $(\mathrm{n}=196)$

Estudo de coorte prospectivo Estudantes de pósgraduação em medicina $(n=63)$

Estudo qualitativo. Estudantes de graduação em medicina $(n=120)$
Alto

Moderado

Moderado

Alto

Alto

Moderado

Moderado

Alto

Moderado

Moderado

Alto

Moderado

Alto

Alto

Moderado

Moderado

Moderado

Alto

Moderado 
Zhang et al., 2019. A flipped classroom method based on a small private online China course in physiology

Cabrera et al.,

2019

EUA

Borit \&

Stangbaltaite-

Mouhat, 2020.

Noruega

Halasa et al., 2020. Comparing student achievement in traditional learning with a EUA

Zheng \& Zhang, 2020. EUA

Kolahdouzan et al., 2020. Irã

Arya et al., 2020. Índia combination of blended and flipped learning

Self-regulated learning: the effect on medical student learning outcomes in a flipped classroom environment

The effect of case-based teaching and flipped classroom methods in comparison with lecture method on learning and satisfaction of internship students in surgery

Flipped classroom versus traditional lecture in training undergraduates in pediatric epilepsy

Kohler et al., 2020. Flipping the Classroom in Otolaryngology Residencies EUA

Kurup et al., 2020. The Feasibility of Incorporating a Flipped Classroom Model in Índia an Anesthesia Residency Curriculum-Pilot Study

Herrero et al., 2020. Espanha

DePietro et al., 2021. EUA

Zheng et al., 2019. Self-regulated learning in a competency-based and flipped EUA learning environment: learning strategies across achievement levels and years

Khoury et al., 2020. Canadá

Shah et al., 2020. EUA

Wu et al., 2020

China

Yang et al., 2020.

China

Preclerkship Point-of-Care Ultrasound: Image Acquisition and Clinical Transferability

Efficacy of quality improvement and patient safety workshops for students: a pilot study

Existing contradictions and suggestions: flipped classroom in radiology courses of musculoskeletal disease under Chinese medical educational mode from medical imaging student perspective

Flipped classroom combined with human anatomy web-based learning system shows promising effects in anatomy education

Huang et al., 2020. Effects of a quasi-experimental study of Taiwan using flipped classroom approach to teach evidence-based medicine to medical technology students
Estudo prospectivo. Estudantes de graduação em medicina $(\mathrm{n}=197)$

Estudo prospectivo randomizado. Estudantes de pós-graduação em medicina $(\mathrm{n}=40)$

Estudo misto. Estudantes de graduação em odontologia $(n=102)$

Estudo prospectivo randomizado. Estudantes de graduação em enfermagem $(n=152)$

Estudo misto. Estudantes de graduação em medicina $(n=146)$

Estudo prospectivo randomizado. Estudantes de pós-graduação em medicina $(n=136)$

Estudo prospectivo comparativo. Estudantes de graduação em medicina $(\mathrm{n}=60)$

Estudo prospectivo randomizado. Estudantes de pós-graduação de cursos da área da saúde $(\mathrm{n}=107)$

Estudo misto. Estudantes de pós-graduação de cursos da área da saúde $(\mathrm{n}=10)$

Relato de experiência. Estudantes de graduação em medicina $(n=430)$

Relato de experiência. Estudantes de graduação em medicina $(n=10)$

Estudo qualitativo. Estudantes de graduação em medicina $(n=26)$

Estudo prospectivo. Estudantes de pós-graduação em medicina $(n=32)$

Estudo prospectivo. Estudantes de graduação de cursos da saúde $(\mathrm{n}=146)$

Estudo qualitativo. Estudantes de graduação em radiologia $(n=55)$

Estudo qualitativo. Estudantes de graduação em medicina

$(\mathrm{n}=89)$

Estudo prospectivo randomizado. Estudantes de graduação em medicina $(n=62)$

Alto

Estudo qualitativo. Estudantes de pós-graduação

Moderado

Moderado

Alto

Moderado

Alto

Moderado

Alto

Alto

Alto

Moderado

Baixo

Baixo

Moderado

Moderado

Moderado

Moderado

Moderado

em medicina $(n=44)$

Zante et al., 2020. Physiology education for intensive care medicine residents: A Suíça

15-minute interactive peer-led flipped classroom session 
Já o Quadro 1 descreve as principais estratégias de ensino-aprendizagem encontradas nos estudos. Observou-se que aspectos como o interesse e satisfação dos estudantes envolvidos, o desenvolvimento de troca colaborativa de conhecimento, habilidades de comunicação e argumentação, o posicionamento do estudante no centro do processo ensino-aprendizagem, a autonomia do estudante para assumir o controle de uma aprendizagem autodirigida (Andrés et al., 2018; Melo Júnior et al., 2018; Menegaz et al., 2018; Katz et al., 2017; Kohli et al., 2019), a melhoria na relação entre professor-aluno (Andrés et al., 2018; Cho \& Kim, 2019), a melhoria no desempenho dos alunos em atividades propostas e a ampliação do pensamento clínico de forma crítica (Tang et al., 2017) foram aspectos que mostraram uma positiva relação com a utilização destas estratégias diante da sala de aula invertida.

Quadro 1. Principais estratégias de ensino-aprendizagem utilizadas nos estudos selecionados. Recife-PE, 2020.

\begin{tabular}{|c|c|}
\hline $\begin{array}{l}\text { Estratégias de Ensino- } \\
\text { Aprendizagem }\end{array}$ & Estudos \\
\hline $\begin{array}{l}\text { Vídeos educativos online } \\
\quad \text { (videoaulas) }\end{array}$ & $\begin{array}{l}\text { Oliveira et al., 2018; Melo Júnior et al., 2018; Menegaz et al., 2018; Tang et al., 2017; Marco et al., 2017; Jesurasa et al., 2017; } \\
\text { Rui et al., 2017; Fleagle et al., 2018; Martinelli et al., 2017; Riddell et al., 2017; Cheng et al., 2016; Hsu et al., 2016; Lew, 2016; } \\
\text { Ferrer-Torregrosa et al., 2016; Lochner et al., 2016; Green \& Schlairet, 2017; Ihm et al., 2017; Ridell et al., 2016; Gopalan \& } \\
\text { klann, 2017; King et al., 2017; Philips et al., 2017; Fátima et al., 2017; Kim et al., 2018; Seo et al., 2018; Wu et al., 2018; Jung, } \\
\text { 2018; Lee \& Kim, 2018; Kohli et al., 2019; Cho \& Kim, 2019; Chu, et al., 2018; Roe et al., 2019; He et al., 2019; Kühl et al., } \\
\text { 2019; Sohn et al., 2019; Zhang et al., 2019; Cabrera et al., 2019; Borit \& Stangbaltaite-Mouhat, 2020; Halasa et al., 2020; Arya } \\
\text { et al., 2020; Herrero et al., 2020; DePietro et al., 2021; Zheng et al., 2020; Wu et al., 2020. }\end{array}$ \\
\hline $\begin{array}{l}\text { Atividades em grupo (exercícios, } \\
\text { debates, fóruns de discussão e } \\
\text { outros). }\end{array}$ & $\begin{array}{l}\text { Oliveira et al., 2018; Melo Júnior et al., 2018; Menegaz et al., 2018; Andrés, 2018; Domínguez et al., 2015; Tang et al., 2017; } \\
\text { Hsu et al., 2016; El-Banna et al., 2017; Ihm et al., 2017; Nishigawa et al., 2017; Lossius et al., 2016; Mc Lean et al., 2016; } \\
\text { Gopalan \& klann, 2017; Fátima et al., 2017; Kim et al., 2018; Xião et al., 2018; Jung, 2018; Kohli et al., 2019; Cho \& Kim, } \\
\text { 2019; Roe et al., 2019; Borit \& Stangbaltaite-Mouhat, 2020; Halasa et al., 2020; Zheng \& Zhang, 2020; Zheng et al., 2019; Shah } \\
\text { et al., 2020; Yang et al., 2020; Sajid et al., 2016. }\end{array}$ \\
\hline $\begin{array}{l}\text { Materiais de leitura / atividades } \\
\text { assíncronas antes do encontro } \\
\text { síncrono (online) ou presencial em } \\
\text { sala de aula (artigos, livros e outros) }\end{array}$ & $\begin{array}{l}\text { Oliveira et al., 2018; Melo Júnior et al., 2018; Menegaz et al., 2018; Andrés 2018; Domínguez et al., 2015; Tang et al., 2017; } \\
\text { Jesurasa et al., 2017; Rui et al., 2017; Cheng et al., 2016; Green \& Schlairet, 2017; Ridell et al., 2016; King et al., 2017; Lee \& } \\
\text { Kim, 2018; Cho \& Kim, 2019; Graham et al., 2019; Sohn et al., 2019; Cabrera et al., 2019; Kohler et al., 2020; DePietro, et al., } \\
\text { 2021; Zheng et al., 2019; Khoury et al., 2020; Wu et al., 2020; Zante et al., 2020; Lochner et al., 2016; Martinelli, 2018; Jung, } \\
\text { 2018; Lew, 2016. }\end{array}$ \\
\hline $\begin{array}{l}\text { Apresentação/resolução/discussão } \\
\text { de casos (estudos de caso) }\end{array}$ & $\begin{array}{l}\text { Oliveira et al., 2018; Bas-Sarmiento et al., 2017; Rui et al., 2017; Riddell et al., 2017; Lin et al., 2017; Tan et al., 2015; Bossaer } \\
\text { et al., 2016; Mc Lean et al., 2016; Gillispie, 2016; Fátima et al., 2017; Petit et al., 2017; Martinelli, 2018; Xião et al., 2018; Cho } \\
\text { \& Kim, 2019; Salabe \& Chowdary, 2019; Kolahdouzan et al., 2020; Huang et al., 2020. }\end{array}$ \\
\hline $\begin{array}{l}\text { Atividades de avaliação de } \\
\text { conhecimentos (questionários, pré e } \\
\text { pós-testes, formulários avaliativos, } \\
\text { perguntas e respostas, feedback e } \\
\text { outros) }\end{array}$ & $\begin{array}{l}\text { Lucardie et al., 2017; Bonnes et al., 2017; Carrick et al., 2017; Lin et al., 2017; Lew, 2016; Sajid et al., 2016; Lochner et al., } \\
\text { 2016; Mc Lean et al., 2016; Wu et al., 2018; Beom et al., 2018; Lee \& Kim, 2018; Sohn et al., 2019; Kohler et al., 2020; Wu et } \\
\text { al., 2020; Zante et al., 2020. }\end{array}$ \\
\hline $\begin{array}{l}\text { Materiais audiovisuais (figuras, } \\
\text { gráficos visuais, mapas } \\
\text { mentais/conceituais, materiais de } \\
\text { áudio, podcasts, palestras } \\
\text { previamente gravadas e outros) }\end{array}$ & $\begin{array}{l}\text { Fleagle et al., 2018; Fátima, et al., 2017; Herrero et al., 2020; Bossaer et al., 2016; Boysen-Osborn et al., 2016; Petit et al., 2017; } \\
\text { Kurup et al., 2020; Wu et al., 2020; Yang et al., 2020; Ferrer-Torregrosa et al., 2016; Lin et al., } 2017 .\end{array}$ \\
\hline $\begin{array}{l}\text { Módulos de conteúdo em } \\
\text { ambientes/plataformas virtuais de } \\
\text { aprendizagem }\end{array}$ & Bonnes et al., 2017; Carrick et al., 2017; Zhang et al., 2019; Yang et al., 2020. \\
\hline $\begin{array}{l}\text { Aprendizagem autodirigida (estudo } \\
\text { individual de forma assíncrona) }\end{array}$ & Girgis \& Miller 2018; Tan et al., 2015; Bossaer et al., 2016. \\
\hline
\end{tabular}




\begin{tabular}{|c|l|}
\hline $\begin{array}{c}\text { Aprendizagem baseada em } \\
\text { problemas }\end{array}$ & Gillispie, 2016; Graham et al., 2019. \\
\hline $\begin{array}{c}\text { Aprendizagem baseada em equipes } \\
\text { (Team-Based Learning) }\end{array}$ & Oliveira et al., 2018; Melo Júnior et al., 2018; Menegaz et al., 2018; Gopalan \& klann, 2017. \\
\hline $\begin{array}{c}\text { Aprendizagem baseada em } \\
\text { Simulação (simulações clínicas, } \\
\text { realidade virtual) }\end{array}$ & Martinelli, 2018; Ferrer-Torregrosa et al., 2016. \\
\hline $\begin{array}{c}\text { Palestras/aulas interativas online } \\
\text { (síncronas) }\end{array}$ & Sajid et al., 2016; Bossaer et al., 2016; Mc Lean et al., 2016; Gillispie, 2016. \\
\hline Palestras presenciais e workshops & Zhang et al., 2019; Gopalan et al., 2018. \\
\hline Atividades práticas em laboratório & Katz et al., 2017. \\
\hline
\end{tabular}

Fonte: Dados da pesquisa.

Com relação às limitações, fragilidades e dificuldades encontradas na aplicação da sala de aula invertida, foi evidenciada ausência da utilização desta estratégia em alguns ambientes de aprendizagem pelos docentes e instituições, além de mais carga de trabalho, maior responsabilidade para o aluno ao gerenciar seu tempo de aprendizagem e necessidade de altos níveis de motivação (Dominguez, et al., 2015; Marco, et al., 2017; Lucardie, et al., 2017).

Por fim, na Tabela 2 encontram-se destacadas as principais contribuições da sala de aula invertida no processo de ensino-aprendizagem na formação superior em saúde.

Tabela 2. Contribuições da sala de aula invertida no ensino superior em saúde. Recife - PE, 2020 (n=77).

Título do estudo
$\begin{aligned} & \text { Team-Based Learning como forma de aprendizagem colaborativa e } \\ & \text { sala de aula invertida com centralidade nos estudantes no processo } \\ & \text { ensino-aprendizagem }\end{aligned}$
Sala de aula invertida para o ensino do conteúdo abertura coronária
em Endodontia
Flipped Classroom no ensino de gerenciamento em
enfermagem: relato de experiência
Impacto académico de una estrategia de salón invertido en
Anatomía
Impacto de la estrategia de aula invertida en el ambiente de
aprendizaje en cirugía: una comparación con la clase magistral
in ophthalmology clerkship

Does the acceptance of hybrid learning affect learning approaches in France?

\section{Contribuições da sala de aula invertida no ensino superior em saúde}

Permitiu que os estudantes exercitassem suas habilidades de comunicação, argumentação e convencimento, melhorando sua interação entre pares, com os docentes e desempenho pessoal.

Contribuiu na autoavaliação de aquisição de competências, onde houve aprendizado significativo pelos estudantes e aumento da motivação. A estratégia foi adequada para o estudo do conteúdo abordado.

Foi possível que os estudantes se posicionassem, argumentassem e se relacionassem a partir de suas experiências prévias, ampliando seu entendimento sobre os conteúdos ministrados.

A estratégia demonstrou ter um efeito importante na condução de discussões, projetos em pequenos grupos, na aprendizagem individual e na melhoria da interação alunoprofessor.

Apresentou impacto positivo na construção do pensamento crítico dos estudantes. A pontuação nos domínios avaliados pelo estudo foram mais altas com a estratégia de sala de aula invertida.

Ajudou a promover sua motivação dos alunos para o aprendizado, melhorar a compreensão dos materiais do curso e aprimorar as habilidades de comunicação e pensamento clínico.

A utilização da sala de aula invertida ajudou na aceitação dos alunos pelo modelo de aprendizagem híbrido (aulas presenciais e aulas online), devido à flexibilidade da estratégia.

Efficacy of empathy training in nursing students: A quasiexperimental study

O treinamento utilizando a estratégia da sala de aula invertida mostrou-se eficaz para melhorar a empatia dos estudantes universitários, onde estes adquiriram competências sobre o assunto. 
What factors facilitate the engagement with flipped classrooms used in the preparation for postgraduate medical membership examinations?

Teaching procedural skills to medical students: A pilot procedural skills lab

Friend or Foe? Flipped Classroom for Undergraduate Electrocardiogram Learning: a Randomized Controlled Study

Flipping the classroom to teach Millennial residents medical leadership: a proof of concept

Flipping the Quality Improvement Classroom in Residency Education

Application of flipped classroom pedagogy to the human gross anatomy laboratory: Student preferences and learning outcomes

Implementation of a "Flipped Classroom" for Neurosurgery

Resident Education

Results of a Flipped Classroom Teaching Approach in Anesthesiology Residents

Does the Flipped Classroom Improve Learning in Graduate Medical Education?

Randomized Controlled Study of a Remote Flipped Classroom Neuro-otology Curriculum

Facing the challenges in ophthalmology clerkship teaching: Is flipped classroom the answer?

The "flipped classroom" approach: Stimulating positive learning attitudes and improving mastery of histology among medical students

An Investigation of the Outcomes of PGY Students' Cognition of and Persistent Behavior in Learning through the Intervention of the Flipped Classroom in Taiwan

Creating a contemporary clerkship curriculum: the flipped classroom model in emergency medicine

Can blended learning and the flipped classroom improve student learning and satisfaction in Saudi Arabia?

Distance learning ects and flipped classroom in the anatomy learning: comparative study of the use of augmented reality, video and notes

Combining traditional anatomy lectures with e-learning activities how do students perceive their learning experience?

Acceptability of the flipped classroom approach for in-house teaching in emergency medicine

Flipping around the classroom: Accelerated Bachelor of Science in Nursing students' satisfaction and achievement

Moving toward heutagogical learning: Illuminating undergraduate nursing students' experiences in a flipped classroom
Possibilitou a variação nos estilos de aprendizagem e ensino de indivíduos, bem como a viabilidade e flexibilidade do design geral do curso.

Permitiu que os alunos se familiarizassem com os aspectos práticos da execução de habilidades processuais sem a necessidade de recursos para professores ou sala de aula.

Permitiu um aprendizado personalizado, possibilitando que os alunos com diferentes hábitos e habilidades de aprendizado gerenciassem seus ritmos de aprendizado de acordo com sua própria situação específica.

Possibilitou o alcance de altos níveis de interação, a utilização ideal do tempo do aluno e do professor/especialista e a aplicação direta do conhecimento no ambiente de prática.

A sala de aula invertida aumentou significativamente a pontuação dos alunos em comparação àqueles que não foram expostos a ela, bem como permitiu o maior envolvimento dos alunos nos módulos online.

Melhorou a preparação do aluno para as atividades no laboratório, oportunizou atividades integrativas que conectaram o laboratório e o material de aula e explorou as correlações clínicas entre os assuntos.

Permitiu um maior envolvimento e desempenho aprimorado ao utilizar ferramentas de avaliação de conhecimento validadas.

Resultou em melhorias na retenção de conhecimento e foi a preferida pelos estudantes em comparação à abordagem tradicional.

Houve diferenças significativas na melhoria do desempenho para determinados testes na utilização da sala de aula invertida.

Complementa o ensino tradicional a partir de ferramentas de baixo custo e melhora o ambiente de aprendizagem.

Ajudou os alunos em habilidades como resolução de problemas, pensamento criativo trabalho em equipe, utilizando melhor o tempo em sala de aula para desenvolver habilidades cognitivas.

Aumentou significativamente a pontuação dos estudantes nos testes, onde os alunos se beneficiaram da sala de aula invertida.

Permitiu a mudança no comportamento da aprendizagem passiva para a aprendizagem ativa.

Permitiu a participação ativa nas atividades e a síntese adequada do conhecimento. Os alunos avaliaram que a interatividade, a discussão e a tomada de decisão são vantagens desse formato

Mostrou resultados promissores em relação à maior satisfação dos alunos. Permitiu a substituição da leitura passiva por um aprendizado ativo, que aprimora o pensamento crítico e a retenção de informação.

Aumentou os conhecimentos adquiridos pelos estudantes e aprimorou o aprendizado autônomo destes em sua formação acadêmica.

Promoveu a aprendizagem ativa durante as aulas, apoiou os alunos na distribuição dos estudos e os aproximou do material didático, bem como melhorou a estrutura do curso ofertado.

Contribuiu com a satisfação do aluno em aspectos como discussão baseada em casos, interação com colegas, aplicação de conhecimento, aprendizagem autodirigida e aprendizagem em pequenos grupos.

Preparou os alunos para o sucesso nos exames regulares, aumentando significativamente a pontuação.

Ofereceu possibilidades promissoras para facilitar o envolvimento dos alunos na aprendizagem, bem como revelou sentimentos positivos dos alunos sobre a estratégia. 
Flipped-learning course design and evaluation through student selfassessment in a predental science class

Comparison between flipped classroom and team-based learning in fixed prosthodontic education

Student Performance in a Pharmacotherapy Oncology Module Before and After Flipping the Classroom

Low Back Pain in the Emergency Medicine Department: A Flipped Classroom Module

An Interprofessional Small-Group Learning Experience in Early Childhood Caries With Fluoride Varnish Application

Flipping the Advanced Cardiac Life Support Classroom with Teambased Learning: Comparison of Cognitive Testing Performance for Medical Students at the University of California, Irvine, United States

Flipped classrooms and student learning: not just surface gains

Using the Flipped Classroom to Bridge the Gap to Generation Y

The effect of flipped teaching combined with modified team-based learning on student performance in physiology

Replacing Lectures with Small Groups: The Impact of Flipping the Residency Conference Day

Video-Based Teaching of Image-Guided Breast Interventions: Stereotactic Core Biopsy Using a Prone Table

Flipped classroom instructional approach in undergraduate medical education

What millennial medical students say about flipped learning

The relationship between non-cognitive student attributes and academic achievements in a flipped learning classroom of a predental science course

Dental students' learning attitudes and perceptions of YouTube as a lecture video hosting platform in a flipped classroom in Korea

Utilization of the Flipped Classroom in Anesthesiology Graduate Medical Education: An Initial Survey of Faculty Beliefs and Practices About Active Learning

Flipped classroom narrows the performance gap between low- and high-performing dental students in physiology

Embracing the flipped classroom: the planning and execution of a faculty workshop

Helps from flipped classroom in learning suturing skill: The medical students' perspective

Analysis of satisfaction and academic achievement of medical students in a flipped class
Aumentou a preparação dos alunos antes da aula presencial e melhorou as habilidades de discussão em grupo e satisfação com as atividades.

Aumentou as pontuações nos resultados dos exames finais.

Promove abordagens de aprendizagem ativa na sala de aula

Houve aumento imediato no conhecimento. Foi notado que era útil para os alunos terem uma explicação prévia do formato para que eles soubessem o que esperavam e tivessem algum conforto com o formato.

Promoveu discussões de caso em grupo mais valiosas e um melhor uso do tempo de aula alocado. Os alunos avaliaram os materiais preparatórios para estudar antes da aula como o fator mais eficaz.

A combinação da estratégia de sala de aula invertida com a aprendizagem baseada em equipe no programa educacional do curso melhorou os resultados dos testes escritos.

Permitiu que os alunos apresentassem um alto índice de conhecimento acerca do seu próprio processo de aprendizagem, e que pudessem mudar de estratégia conforme necessário e gerenciar seu tempo.

Aumentou o envolvimento dos alunos com o seu aprendizado e os levou a uma melhor retenção do conhecimento.

O ensino invertido melhorou o desempenho dos alunos em $17,5 \%$, mudando a forma como eles normalmente estudavam e preparando-os para a aula presencial.

Aumento significativamente maior no tempo gasto com aprendizagem externa (livros didáticos, recursos de aprendizagem online e periódicos) na preparação para as discussões em grupo.

Permitiu que os alunos demonstrassem uma maior compreensão do assunto e um melhor planejamento da técnica, além de conforto com o procedimento.

Possibilitou uma maior interação em sala de aula a partir do conhecimento prévio, além de tornar o aprendizado divertido, segundo os estudantes. Os questionários ajudaram a consolidar o conhecimento.

Quanto ao ritmo, a sala de aula invertida acelera o aprendizado. A depender do conteúdo, eles são melhores ensinados por meio de atividades no formato invertido. Dá ao estudante a possibilidade de estudar no seu próprio ritmo e quando estiver no seu melhor.

Melhorou o envolvimento dos estudantes nas discussões em grupo.

A inserção de tecnologias digitais durante a sala de aula invertida promoveu a compreensão dos alunos e o aprendizado interativo.

Permitiu a utilização de uma grande variedade de materiais e atividades que podem ser utilizadas e disponibilizadas.

Fortaleceu a colaboração entre os alunos nos trabalhos em equipe, melhorou o entendimento do conteúdo ministrado, aumentou o desempenho dos alunos nos questionários

Permite que os professores atuem como guias e que os alunos assumam um papel mais responsável e ativo em sua aprendizagem.

Contribuiu para a aquisição de novas habilidades, foi uma prática bem aceita pelos acadêmicos e contribuiu positivamente para a inovação na medicina.

Auxiliou na interação entre os estudantes e tornou a colaboração em classe mais eficaz. Os alunos estavam satisfeitos com a sala de aula invertida e que ela foi útil para a aprendizagem autodirigida. 
Flipped-classroom training in advanced cardiopulmonary life support

Enhancement of student perceptions of learner-centeredness and community of inquiry in flipped classrooms

Dental education: Lecture versus flipped and spaced learning

Outcomes and influential factors applying flipped learning methods in a clinical adult nursing practicum

The effects of the flipped classroom in teaching evidence based nursing: A quasi-experimental study

Learning with technology in physiotherapy education: design, implementation and evaluation of a flipped classroom teaching approach

Flipped classroom - An innovative teaching model to train undergraduate medical students in community medicine

The effects of flipped classrooms on undergraduate pharmaceutical marketing learning: A clustered randomized controlled study

Investigating the self-study phase of an inverted biochemistry classroom - collaborative dyadic learning makes the difference

Effect of a Flipped Classroom on Knowledge Acquisition and Retention in an Internal Medicine Residency Program

The flipped classroom model for an undergraduate epidemiology course

A flipped classroom method based on a small private online course in physiology

The flipped-classroom approach to teaching horizontal strabismus in ophthalmology residency: a pilot study

GoDental! Enhancing flipped classroom experience with gamebased learning

Comparing student achievement in traditional learning with a combination of blended and flipped learning

Self-regulated learning: the effect on medical student learning outcomes in a flipped classroom environment

The effect of case-based teaching and flipped classroom methods in comparison with lecture method on learning and satisfaction of internship students in surgery

Flipped classroom versus traditional lecture in training undergraduates in pediatric epilepsy

Flipping the Classroom in Otolaryngology Residencies

The Feasibility of Incorporating a Flipped Classroom Model in an Anesthesia Residency Curriculum-Pilot Study
Promoveu a participação mais ativa dos estudantes e aumentou sua capacidade de compreensão dos assuntos.

Promoveu um ambiente de aprendizagem centrado no aluno e permitiu o desenvolvimento de habilidades sociais e cognitivas entre os estudantes.

Aumentou os ganhos de conhecimento, reforçou o pensamento crítico, inspirou o aprendizado de coisas novas, melhorou a capacidade de concentração e discussão sobre o assunto.

Aumentou as interações professor-aluno e mostrou-se eficaz em práticas clínicas.

Aumentou o acúmulo de conhecimento dos alunos e a autoeficácia destes na prática clínica.

Melhorou o desempenho dos alunos no programa. Estes responderam positivamente ao ambiente de aprendizagem colaborativa, especialmente em relação à autonomia e flexibilidade associadas.

Aumentou a aprendizagem e permitiu a aplicação do conhecimento teórico na prática. Houve feedback positivo para o ensino em sala de aula invertida.

Melhorou o desempenho dos alunos e gerou uma interação maior entre discente e docente em comparação ao método tradicional.

Foi percebido diferenças no sucesso da aprendizagem, dependendo do material de aprendizagem fornecido. A aprendizagem didática colaborativa levou a um melhor resultado de aprendizagem.

Garantiu um maior tempo dedicado às atividades a serem realizadas presencialmente na sala de aula, além de aumentar a satisfação e desempenho dos residentes do programa.

Aprimorou a aprendizagem autodirigida e permitiu o alcance de melhores resultados de aprendizagem. O tempo gasto no estudo do material didático antes da aula aumentou significativamente.

Aprimorou a capacidade de compreensão por meio da discussão em grupo, aumentou a satisfação com o curso e contribuiu com a capacidade de autoaprendizagem.

Permitiu o acesso prévio ao conteúdo da aula. Para os alunos, poder pausar, repetir e avançar os conteúdos no componente online são diferenciais da estratégia durante o processo de aprendizagem.

Aumentou o envolvimento e integração dos alunos, a motivação, a concentração e os ajudou aprender mais sobre o assunto. Houve uma correlação positiva entre o grau de diversão e concentração.

Aumentou significativamente os níveis de conhecimento e satisfação com a aprendizagem.

Foi estatisticamente útil na fase de monitoramento do autoaprendizado como estratégia de aprendizagem.

Aumentou a qualidade da aprendizagem, proporcionou um dinamismo maior nas atividades, favoreceu a motivação em estudar o assunto proposto e levou à obtenção de pontuações maiores.

Resultou em melhores pontuações em relação ao método tradicional de ensino para o treinamento de graduandos em medicina. Os alunos obtiveram melhores desempenhos na gestão da temática trabalhada.

Permitiu aprender o conteúdo principal antes das sessões de aula, aprimora a aprendizagem, permite a discussão em grupo do conteúdo principal, permite a combinação da aprendizagem presencial e online.

Foi percebido uma melhora no aprendizado dos alunos e em seu envolvimento nas atividades. 
Flipped classroom improves results in pathophysiology learning:

Medical Student Education During the COVID-19 Pandemic: Initial Experiences Implementing a Virtual Interventional Radiology Elective Course

Self-regulated learning in a competency-based and flipped learning environment: learning strategies across achievement levels and years

Preclerkship Point-of-Care Ultrasound: Image Acquisition and

Efficacy of quality improvement and patient safety workshops for students: a pilot study results of a nonrandomized controlled study Clinical Transferability

Facilitou a aprendizagem fora e dentro da sala de aula. Também aumentou o aprendizado ativo, o que resultou em maiores ganhos de aprendizagem.

O fornecimento de materiais de leitura e vídeo de pré-aprendizagem permitiu que os facilitadores se concentrassem em esclarecer conceitos difíceis e se envolver com os alunos durante as aulas virtuais.

Estratégias entre pares utilizadas na sala de aula invertida, como trabalhar com colegas de classe para concluir tarefas ou discutir os materiais com um grupo de alunos, contribuíram com a aprendizagem.

Promoveu um ambiente mais confortável e colaborativo, transmitiu de forma eficaz o conhecimento, padronizou o conteúdo educacional e aumentou a acessibilidade, independentemente da localização.

O ritmo personalizado, como um dos aspectos de aprendizagem da sala de aula invertida, permitiu que os alunos organizassem sua vida em torno de um determinado cronograma

Proporcionou ao aluno melhores experiências de aprendizagem. O tempo de discussão e comunicação aluno-professor aumentaram, e as perguntas dos alunos foram resolvidas de forma satisfatória.

Melhorou a aprendizagem autônoma dos alunos, aumentou o interesse deles no aprendizado de anatomia humana e permitiu o uso de atividades interativas.

\section{web-based learning system shows promising}

Flipped classroom combined with human anatomy

effects in anatomy education

Effects of a quasi-experimental study of using flipped classroom approach to teach evidence-based medicine to medical

technology students

Physiology education for intensive care medicine residents: A 15 minute interactive peer-led flipped classroom session
Permitiu que os alunos colocassem o conhecimento em prática, aumentando sua eficácia de aprendizagem A existência de um feedback imediato possibilitou que as perguntas dos alunos fossem resolvidas de forma mais eficaz durante a aula.

A discussão e interação presentes na sala de aula invertida promoveram a compreensão dos conteúdos e facilitou a aplicação do conhecimento em sua prática diária.

Fonte: Dados da pesquisa.

\section{Discussão}

A sala de aula invertida é uma modalidade de e-learning na qual o conteúdo e as instruções são estudados online antes do aluno frequentar a sala de aula, que agora passa a ser o local para trabalhar os conteúdos já estudados, realizando atividades práticas como resolução de problemas e projetos, discussão em grupo, laboratórios etc. Nesta abordagem, ao realizar o estudo prévio de conteúdos, o aluno estará desenvolvendo uma aprendizagem ativa e construindo seu conhecimento, onde o professor trabalhará atuando na resolução das dificuldades dos alunos, ao invés de focar nas apresentações sobre o conteúdo da disciplina. Além disso, os alunos são encorajados a progredir além da compreensão da teoria para adquirirá também habilidades cognitivas, como avaliação e aplicação do conhecimento na prática (Gillispie, 2016; Andrés et al., 2018; Dominguez et al., 2015; Tang et al., 2017; Bonnes et al., 2017).

Em aulas tradicionais, um breve momento de distração do estudante durante a exposição do professor pode ser suficiente para dificultar uma compreensão adequada de alguma explicação. Em contrapartida, na Sala de Aula Invertida, o aluno, em casa, estuda em seu próprio ritmo, tendo a opção de pausar o vídeo e reproduzi-lo quantas vezes achar necessário ou, em caso de textos, reler diversas vezes o que não compreendeu (Mc Lean, et al., 2016). Em caso de dúvida, o aluno tem a possibilidade de recorrer a outras fontes de informações (e.g. páginas da internet, vídeos, livros, etc) (Gopalan \& Klann, 2017). Um estudo realizado na Colômbia e nos Estados Unidos apontam, ainda, que este método contribui na formação do senso crítico do aluno, possibilitando que o aluno participe de discussões, que contribui na melhoria da interação aluno-professor e no desenvolvimento de suas habilidades. Ademais, percebe-se que o aprendizado ativo aumenta o envolvimento do aluno e pode levar a uma melhor retenção do conteúdo abordado (Andrés et al., 2018; Gillispie, 2016; Beom et al., 2018).

Para o desenvolvimento dessas habilidades, Hsu et al. (2016), em seu estudo, revela aspectos que devem ser adotados 
pelos alunos para que consigam desenvolver a aprendizagem de forma eficaz. Dentre os aspectos citados está a teoria da autoeficácia, que se caracteriza pela confiança, crença e capacidade de uma pessoa em mudar seu comportamento para alcançar um objetivo. Dessa forma, esse aspecto torna-se um desafio para diversos professores no que se refere a utilização da sala de aula invertida, pois a autoeficácia depende de fatores como a atitude e motivação do aluno para que a aprendizagem seja desenvolvida (Hsu et al., 2016).

É válido destacar que o uso da sala de aula invertida se intensificou no ano de 2020, em instituições educacionais de diversos países, diante de um cenário de avanços tecnológicos e contextos como o distanciamento social decorrente da pandemia da COVID-19, superando os impactos educacionais, configurando-se como promoção de uma aprendizagem ativa e centrada no aluno. Os artigos analisados apontam que a metodologia pode ser utilizada por diversas áreas da saúde sejam elas, da enfermagem, fisioterapia, medicina e odontologia, reforçando o ensino multiprofissional. Além disso, ressalta-se a grande utilização da sala de aula invertida nos cursos de pós-graduação (Strictu sensu, Lato sensu e residências), que segundo estudos realizados nos Estados Unidos, isso se dá pelo fato de que essa estratégia de ensino auxilia na retenção de conhecimentos (Martinelli et al, 2017).

A partir de nossos resultados, observou-se que as principais estratégias de ensino-aprendizagem foram a realização de atividades em grupo, a disponibilidade de materiais de leitura ou audiovisuais antes dos encontros presenciais, discussão de casos e a atividades para avaliar a aquisição de conhecimentos após a sala de aula invertida. Lucardie et al. (2017) afirma que a utilização de estratégias está atrelada às necessidades e expectativas educacionais, que variam dentro de várias gerações e exigem novas modalidades de ensino. Sendo assim, a escolha de estratégias de ensino-aprendizagem deve ser orientada não apenas pelos valores docentes, mas também por seu alinhamento com as características e preferências dos alunos (Youhasan et al., 2021).

Além destas estratégias, uma das principais ferramentas utilizadas na sala de aula invertida foi a disponibilidade de vídeos educativos online a partir de tecnologias digitais da informação e comunicação. Seo et al. (2018) demonstram em seu estudo que a maioria dos alunos assistiam sempre a vídeos antes da aula, utilizando seus smartphones para isso. A maioria dos alunos avaliou que as videoaulas são mais fáceis de entender do que as aulas presenciais. O estudo indicou que o YouTube é uma plataforma aplicável para oferecer aulas em vídeo e expor os alunos a maiores oportunidades de aprendizagem antes da discussão em sala de aula. Isto corrobora com o avanço tecnológico no ensino em saúde, o que deve ser considerado em se tratando do planejamento de abordagens que atendam às tecnologias de comunicação avançadas e os métodos de aprendizagem, sabendo-se que a estratégia de sala de aula invertida é uma metodologia educacional que está presente na geração atual (Youhasan et al., 2021).

Como os resultados deste estudo apontaram, a utilização da estratégia da sala de aula invertida tem grandes contribuições do cenário do ensino superior em saúde, especialmente pela sua capacidade de aumentar os resultados de aprendizagem dos alunos nas atividades avaliativas, de aumentar a compreensão dos assuntos, de fornecer preparação prévia para as aulas presenciais, de permitir o uso de uma grande variedade de recursos tecnológicos e materiais, bem como de possibilitar a realização de atividades didáticas interativas. Isto corrobora com a percepção de Bordes et al (2021), onde afirma que, entre os objetivos da sala de aula invertida, estão o aumento da preparação pré-aula do aluno e a promoção do envolvimento do aluno com o conteúdo através de seus diversos recursos. Adicionalmente, a sala de aula invertida promove a aprendizagem ativa e incentiva a responsabilidade do aluno pela aprendizagem (Sajid et al., 2020).

Um estudo experimental com estudantes de enfermagem revelou que a utilização da sala de aula invertida, uma vez que pode combinar atividades online diversas e encontros presenciais, torna-se positiva em comparação ao ensino tradicional, uma vez que, os alunos que vivenciam a aprendizagem combinada apresentaram maior nível de conhecimento e satisfação estatisticamente significativos em relação à administração de habilidades e conhecimentos sobre o conteúdo explorado (Halasa et al, 2020). Tais resultados corroboram com estudos realizados em Taiwan e na Noruega em que apresentam resultados 
significativamente positivos com relação a utilização da aprendizagem combinada (Chu et al, 2018; Roe et al, 2019).

Apesar das contribuições e benefícios da sala de aula invertida no ensino em saúde, evidencia-se alguns desafios como a utilização do aprendizado combinado e o aprendizado online em programas educacionais e sua implementação no currículo educacional. Esses desafios podem ser técnicos (a habilidade dos alunos e educadores em usar a tecnologia com sucesso) e organizacionais (compreensão, incentivo e facilitação do gestor para a implementação do blended learning) (Halasa et al, 2020). Sajid et al. (2020) aponta, como desafio da implementação da sala de aula invertida, que as atividades prévias às aulas devem ser planejadas com cautela para que os alunos não as entendam como fardo adicional. De acordo com Ihm et al. (2017), em seu estudo, alguns alunos podem expressar desconforto com softwares, conexões lentas de Internet e falta de conhecimento tecnológico. Além disso, outro desafio está relacionado às barreiras para a aprendizagem online que incluem as competências psicossociais e afetivas (Tan et al, 2015).

Por fim, é importante ressaltar que o presente estudo apresentou algumas limitações. Primeiramente, o fato de apenas algumas áreas da saúde terem sido incluídas na análise limitou os nossos resultados, visto que outros contextos do ensino superior em saúde também possam ser beneficiados pela aplicação da sala de aula invertida. Em segundo lugar, não houve uma análise comparativa entre a sala de aula invertida, como metodologia ativa, e a metodologia de ensino tradicional, de modo a entender de que forma a primeira estratégia teria ou não melhores benefícios em relação ao ensino estritamente presencial. Diante destas observações, acreditamos que estudos futuros sejam necessários para elucidar estes aspectos e assegurar o adequado planejamento de atividades que se beneficiem do uso da sala de aula invertida.

\section{Conclusão}

Por meio desta revisão sistemática, foi possível identificar as principais publicações sobre a utilização da sala de aula invertida no contexto do ensino superior em saúde, bem como suas contribuições para a formação em saúde e as principais estratégias de ensino-aprendizagem utilizadas. Identificou-se também uma predominância do uso dessa estratégia educacional nos cursos de graduação. Percebe-se, então, que a sala de aula invertida destaca-se pelo seu caráter tecnológico inovador, promovendo a autonomia dos estudantes, o fortalecimento do estudo autodirigido e o desenvolvimento de habilidades teóricas e práticas através da aprendizagem combinada, devendo ser incentivada como metodologia ativa de aprendizagem no ensino superior em saúde.

Embora haja desafios, o estudo mostra que é possível enfrentar as dificuldades através da compreensão sobre essa estratégia de ensino, além de se fazer necessário o emprego de investimentos nas instituições de ensino para a implementação de aspectos da sala de aula invertida no currículo educacional. Sendo assim, faz-se necessário treinamentos voltados para o planejamento das estratégias de ensino-aprendizagem condizentes com as características dos estudantes, para que assim ocorra a aprendizagem centrada no aluno e desenvolvimento de conhecimento a partir do senso crítico. Além disso, é imprescindível a realização de novas pesquisas a fim de explorar a temática e oferecer novos pontos de vista sobre a aplicabilidade da sala de aula invertida nos diversos cursos de ensino superior.

\section{Referências}

Andrés, A. O. R. (2018). Impacto académico de una estrategia de salón invertido en Anatomía. Educación Médica Superior. 61-67.

Arruda, E. P. (2020). Educação remota emergencial: elementos para políticas públicas na educação brasileira em tempos de Covid-19. EmRede - Revista De Educação a Distância, 7(1), 257-275.

Arya, V., Gehlawat, V. K., Rana, R. \& Kaushik, J. (2020). Flipped classroom versus traditional lecture in training undergraduates in pediatric epilepsy. Journal of Family Medicine and Primary Care, 9(9), 4805-4808.

Bacich, L., Neto, A. T. \& Trevisani, F. M. (2015). Ensino híbrido: personalização e tecnologia na educação. Penso. 
Bas-Sarmiento, P., Fernández-Gutiérrez, M., Baena-Baños, M. \& Romero-Sánchez, J. M. (2017). Efficacy of empathy training in nursing students: A quasiexperimental study. Nurse Education Today, 59, 59-65.

Beom, J. H., Kim, J. H., Chung, H. S., Kim, S. M., Ko, D. R. \& Cho, J. (2018). Flipped-classroom training in advanced cardiopulmonary life support. Plos One, 13(9), 1-12.

Bonnes, S. L., Ratelle, J. T., Halvorsen, A. J., Carter, K. J., Hafdahl, L. T., Wang, A. T., Mandrekar, J. N., Oxentenko, A. S., Beckman, T. J. \& Wittich, C. M. (2017). Flipping the Quality Improvement Classroom in Residency Education. Academic Medicine, 92(1), $101-107$.

Bordes, S. J. et al. (2021). Towards the optimal use of video recordings to support the flipped classroom in medical school basic sciences education. Medical Education Online, $26(1), 1841406$.

Borit, M. \& Stangbaltaite-Mouhat, L. (2020). GoDental! Enhancing flipped classroom experience with game-based learning. European Journal of Dental Education, 24(4), 763-772.

Bossaer, J. B., Panus, P., Stewart, D. W., Hagemeier, N. E. \& George, J. (2016). Student Performance in a Pharmacotherapy Oncology Module Before and After Flipping the Classroom. American Journal of Pharmaceutical Education, 80(2), 1-6.

Boysen-Osborn, M., Anderson, C. L., Navarro, R., Yanuck, J., Strom, S., McCoy, C. E., Youm, J., Ypma-Wong, M. F. \& Langdorf M. I. (2016). Flipping the advanced cardiac life support classroom with team-based learning: comparison of cognitive testing performance for medical students at the University of California, Irvine, United States. Journal of Educational Evaluation for Health Professions, 13(11), 1-20.

Brasil. (2012). Diretrizes metodológicas elaboração de revisão sistemática e metanálise de ensaios clínicos randomizados. Editora do Ministério da Saúde.

Cabrera, M. T. et al. (2019). The flipped-classroom approach to teaching horizontal strabismus in ophthalmology residency: a pilot study. Journal of American Association for Pediatric Ophthalmology and Strabismus, 23(4), 1-12.

Carrick, F. R., Abdulrahman, M., Hankir, A., Zayaruzny, M., Najem, K., Lungchukiet, P. \& Edwards, R. A. (2017). Randomized Controlled Study of a Remote Flipped Classroom Neuro-otology Curriculum. Frontiers in Neurology, 8(349), 1-10.

Cheng, X., Lee, K. H., Chang, E. Y. \& Yang, X. (2016). The "flipped classroom" approach: Stimulating positive learning attitudes and improving mastery of histology among medical students. Anatomical Sciences Education, 10(4), 317-327.

Cho, M. K. \& Kim, M.Y. (2019). Outcomes and influential factors applying flipped learning methods in a clinical adult nursing practicum. International Journal of Nursing practice, 25(2), 1-10.

Chu, T. L., Wang, J., Monrouxe, L., Sung, Y. C., Kuo, C. L., Ho, L. H. \& Lin, Y. E. (2018) The effects of the flipped classroom in teaching evidence based nursing: A quasi-experimental study. Plos One, 14(1), 1-12.

DePietro, D. M., Santucci, S. E., Neil E. Harrison, N. E. et al. (2021). Medical Student Education During the COVID-19 Pandemic: Initial Experiences Implementing a Virtual Interventional Radiology Elective Course. Academic Radiology, 28, 128-135.

Domínguez, L. C., Vega, N. V., Espitia, E. L., Sanabria, A. E., Corso, C., Serna, A. M., \& Osorio, C. (2015). Impacto de la estrategia de aula invertida en el ambiente de aprendizaje en cirugía: una comparación con la clase magistral. Biomédica, 35, 513-521.

El-Banna, M. M., Whitlow, M. \& McNelis, A. M. (2017). Flipping around the classroom: Accelerated Bachelor of Science in Nursing students' satisfaction and achievement. Nurse Education Today, 56, 41-46.

Fatima, S. S., Arain, F. M. \& Enam, S. A. (2017). Flipped classroom instructional approach in undergraduate medical education. Pakistan Journal of Medical Sciences, 33(6),1424-1428.

Ferrer-Torregrosa, J., Jiménez-Rodríguez, M. Á., Torralba-Estelles, J., Garzón-Farinós, F., Pérez-Bermejo, M. \& Fernández-Ehrling, N. (2016). Distance learning ects and flipped classroom in the anatomy learning: comparative study of the use of augmented reality, video and notes. BMC Medical Education, 16(1), $1-9$.

Fleagle, T. R., Borcherding, N. C., Harris, J. \& Hoffmann, D. S. (2017). Application of flipped classroom pedagogy to the human gross anatomy laboratory: Student preferences and learning outcomes. Anatomical Sciences Education, 11(4), 385-396.

Gillispie V. (2016). Using the Flipped Classroom to Bridge the Gap to Generation Y. The Ochsner journal, 16(1), 32-36.

Girgis, F. \& Miller, J. P. (2017). Implementation of a "Flipped Classroom” for Neurosurgery Resident Education. Canadian Journal of Neurological Sciences / Journal Canadien des Sciences Neurologiques, 45(1), 76-82.

Gopalan, C., Bracey, G., Klann, M. \& Schmidt, C. (2018). Embracing the flipped classroom: the planning and execution of a faculty workshop. Advances in Physiology Education, 42(4), 648-654.

Gopalan, C. \& klann, M.C. (2017). The effect of flipped teaching combined with modified team-based learning on student performance in physiology. Advances in Physiology Education, 41, 363-367.

Graham, k. L., Cohen, A., Reynolds, E. \& Huang, G. C. (2019). Effect of a Flipped Classroom on Knowledge Acquisition and Retention in an Internal Medicine Residency Program. Journal of Graduate Medical Education, 11(1), 92-97.

Green, R. D. \& Schlairet, M. C. (2017). Moving toward heutagogical learning: Illuminating undergraduate nursing students' experiences in a flipped classroom. Nurse Education Today, 49, 122-128. 
Halasa, S., Abusalim, N., Rayyan, M., Constantino, R. E., Nassar, O., Amre, H., Sharab, M. \& Qadri, I. (2020). Comparing student achievement in traditional learning with a combination of blended and flipped learning. Nursing Open, 7, 1129-1138.

He, Y., Lu, J., Huang, H., He, S., Ma, N., Sha, Z. et al. (2019). The effects of flipped classrooms on undergraduate pharmaceutical marketing learning: A clustered randomized controlled study. Plos one, 14(4), 1-22.

Herrero, J. I. \& Quiroga, J. (2020). Flipped classroom improves results in pathophysiology learning: results of a nonrandomized controlled study. Advances in Physiology Education, 44, 370-375.

Hodges, C., Moore, S., Lockee, B., Trust, T. \& Bond, A. (2020). The Difference between emergency remote teaching and online learning. https://er.educause.edu/articles/2020/3/the-difference-between-emergency-remote-teaching-and-online-learning\#fn 7

Hsu, S.D., Chen, C.J., Chang, W. K. \& Hu, Y.-J. (2016). An Investigation of the Outcomes of PGY Students' Cognition of and Persistent Behavior in Learning through the Intervention of the Flipped Classroom in Taiwan. Plos One, 11(12), 1-14.

Huang, H. L. et al. (2020). Effects of a quasi-experimental study of using flipped classroom approach to teach evidence-based medicine to medical technology students. BMC Medical Education, 20(31), 1-9.

Ihm, J., Choi, H. \& Roh, S. (2017). Flipped-learning course design and evaluation through student self-assessment in a predental science class. Korean Journal of Medical Education, 29(2), 93-100.

Jesurasa, A., Mackenzie, K., Jordan, H. \& Goyder, E. C. (2017). What factors facilitate the engagement with flipped classrooms used in the preparation for postgraduate medical membership examinations? Advances in Medical Education and Practice, 8, 419-426.

Jung, H., An, J. \& Park, K. H. (2018). Analysis of satisfaction and academic achievement of medical students in a flipped class. Korean Journal of Medical Education, 30(2), 101-107.

Katz, L., Finch, A., McKinnish, T., Gilliland, K., Tolleson-Rinehart, S. \& Marks, B. (2017). Teaching procedural skills to medical students: A pilot procedural skills lab. Education for Health, 30(1), 79-83.

Khoury, M., Fotsing, S., Jalali, A., Chagnon, N., Malherbe, S. \& Youssef, N. (2020). Preclerkship Point-of-Care Ultrasound: Image Acquisition and Clinical Transferability. Journal of Medical Education and Curricular Development, 7(1), 1-8.

Kim, M., Sangho Roh, S. \& Jungjoon Ihm, J. (2018). The relationship between non-cognitive student attributes and academic achievements in a flipped learning classroom of a pre-dental science course. Korean Journal of Medical Education, 30(4), 339-346.

King, A. M., Mayer, C., Barrie, M., Greenberger, S. \& Way, D. P. (2018). Replacing Lectures with Small Groups: The Impact of Flipping the Residency Conference Day. Western Journal of Emergency Medicine, 19(1), 11-17.

Kohler, W. J., Favre, N. M., O'Brien, D. C., et al. (2020). Flipping the Classroom in Otolaryngology Residencies. Cureus, 12(7), 1-8.

Kolahdouzan, M., Mahmoudieh, M., Rasti, M., Omid, A., Rostami, A. \& Yamani, N. (2021). The effect of case-based teaching and flipped classroom methods in comparison with lecture method on learning and satisfaction of internship students in surgery. Journal of Education and Health Promotion, 9(1), 1-6.

Kühl, S. J., Schneider, A., Kestler, H. A., Toberer, M., Kühl, M. \& Fischer, M. R. (2019). Investigating the self-study phase of an inverted biochemistry classroom - collaborative dyadic learning makes the difference. BMC Medical Education, 19(64), 01-14.

Kurup, V. \& Sendlewski, G. (2020). The Feasibility of Incorporating a Flipped Classroom Model in an Anesthesia Residency Curriculum-Pilot Study. Yale Journal of Biology and Medicine, 93, 411-417.

Lee, Y. H. \& Kim, K.-J. (2018). Enhancement of student perceptions of learner-centeredness and community of inquiry in flipped classrooms. BMC Medical Education, 18(1), 1-6.

Lew, E. K. (2016). Creating a contemporary clerkship curriculum: the flipped classroom model in emergency medicine. International Journal of Emergency Medicine, 9(25), 1-5.

Lin, Y., Zhu, Y., Chen, C., Wang, W., Chen, T., Li, T., Li, Y., Liu, B., Lian, Y., Lu, L., Zou, Y. \& Liu, Y. (2017). Facing the challenges in ophthalmology clerkship teaching: Is flipped classroom the answer? Plos One, 12(4), 1-14.

Lochner, L., Wieser, H., Waldboth, S. \& Mischo-Kelling, M. (2016). Combining traditional anatomy lectures with e-learning activities: how do students perceive their learning experience? International Journal of Medical Education, 7, 69-74.

Lossius, M., Sposetti, V. \& Black, E. (2016). An interprofessional small-group learning experience in early childhood caries with fluoride varnish application. MedEdPORTAL, 12, 1-6.

Lucardie, A., Berkenbosch, L., Berg, J. V. D \& Busari, J. O. (2017). Flipping the classroom to teach Millennial residents medical leadership: a proof of concept. Advances in Medical Education and Practice, 8, 57-61.

Marco, L. D., Venot, A. \& Gillois, P. (2017). Does the acceptance of hybrid learning affect learning approaches in France? Journal of Educational Evaluation for Health Professions, 14(24) 1-7.

Martinelli, S. M., Chen, F., DiLorenzo, A. N., Mayer, D. C., Fairbanks, S., Moran, K., Ku, C., Mitchell, J. D., Bowe, E. A., Royal, K. D., Hendrickse, A., VanDyke, K. et al. (2017). Results of a Flipped Classroom Teaching Approach in Anesthesiology Residents. Journal of Graduate Medical Education, 9(4), 485490. 
McLean, S., Attardi, S. M., Faden, L. \& Goldszmidt, M. (2016). Flipped classrooms and student learning: not just surface gains. Advances in Physiology Education, 40(1), 47-55.

Melo Júnior, P. M. R., Maia, S. M. A. S., Cunha, R. S. C., Espíndola Júnior, E. \& Bollela, V. R. (2018). Sala de aula invertida para o ensino do conteúdo abertura coronária em Endodontia. Revista da ABENO, 18(2), 182-191.

Menegaz, J. D. C., Dias, G. A. R., Trindade, R. F. S., Leal, S. N. \& Martins, N. K. A. (2018). Flipped Classroom in teaching nursing management: experience report. Escola Anna Nery, 22(3), 1-7.

Narvai, P. C., Mota, A. G., Andrade, F. R. \& Frazão, P. (2018). Saúde Bucal Coletiva e pedagogia da sala de aula invertida: possibilidades e limites no ensino de graduação. Revista da ABENO, 18(1), 124-133.

Nishigawa, K., Omoto, K., Hayama, R., Okura, K., Tajima, T., Suzuki, Y., Hosoki, M., Shigemoto, S., Ueda, M., Rodis, O. M. M., \& Matsuka, Y. (2017). Comparison between flipped classroom and team-based learning in fixed prosthodontic education. Journal of Prosthodontic Research, 61(2), 217-222.

Oliveira, B. L. C. A., Lima, S. F., Rodrigues, L. d. S. \& Pereira Júnior, G. A. (2018). Team-Based Learning como Forma de Aprendizagem Colaborativa e Sala de Aula Invertida com Centralidade nos Estudantes no Processo Ensino-Aprendizagem. Revista Brasileira de Educação Médica, 42(4), 86-95.

Organização Pan-Americana da Saúde (OPAS)/ Organização Mundial da Saúde (OMS). (2020). Histórico da pandemia de COVID-19. https://www.paho.org/pt/covid19/historico-da-pandemia-covid-19

Page, M. J. et al. (2021). The PRISMA 2020 statement: an updated guideline for reporting systematic reviews. https://www.bmj.com/content/372/bmj.n71

Pettit, R., McCoy, L. \& Kinney, M. (2017). What millennial medical students say about flipped learning. Advances in Medical Education and Practice, 8, 487497.

Phillips, J., Sharpe, R. Jr., Sheth M. M. et al. (2017). Video-based teaching of image-guided breast interventions: stereotactic core biopsy using a prone table. MedEdPORTAL, 13, 1-5.

Riddell, J., Jhun, P., Fung, C. C., Comes, J., Sawtelle, S., Tabatabai, R., Joseph, D., Shoenberger, J., Chen, E., Fee, C. \& Swadron, S. P. (2017). Does the Flipped Classroom Improve Learning in Graduate Medical Education? Journal of Graduate Medical Education, 9(4), 491-496.

Riddell, J. C., Sawtelle, S., Jhun, P. et al. (2016). Low back pain in the emergency medicine department: a flipped classroom module. MedEdPORTAL, 12, 1-5.

Roe, Y., Rowe, M., Odegaard, N. B., Sylliaas, H. \& Dahl-Michelsen, T. (2019). Learning with technology in physiotherapy education: design, implementation and evaluation of a flipped classroom teaching approach. BMC Medical Education, 19(291), 1-8.

Rui, Z., Lian-rui, X., Rong-zheng, Y., Jing, Z., Xue-hong, W. \& Chuan, Z. (2017). Friend or Foe? Flipped Classroom for Undergraduate Electrocardiogram Learning: a Randomized Controlled Study. BMC Medical Education, 17(56), 1-9.

Sabale, R. V. \& Chowdary, P. (2019). Flipped Classroom - An Innovative Teaching Model to Train Undergraduate Medical Students in Community Medicine. Education for Health, 32(3), 116-121.

Sajid, M. et al. (2020). Comparative Analysis of Effectiveness Between Flipped Classroom and Lecture-Based Classroom in Undergraduate Medical Education at Alfaisal University. Cureus Journal of Medical Science, 12 (11), e11408.

Sajid, M. R., Laheji, A. F., Abothenain, F., Salam, Y., AlJayar, D. \& Obeidat, A. (2016). Can blended learning and the flipped classroom improve student learning and satisfaction in Saudi Arabia?. International Journal of Medical Education, 7, 281-285.

Santos, C. M. C., Pimenta, C. A. M. \& Nobre, M. R. C. (2007). The PICO strategy for the research question construction and evidence search. Rev. Latino-Am. Enfermagem. 15(3), 508-511.

Schneider, E. I., Suhr, I. R. F., Rolon, V. E. K. \& Almeida, C. M. (2013). Sala de Aula Invertida em EAD: uma proposta de Blended Learning. Revista Intersaberes, 8(16), 68-81.

Seo, C. W., Cho, A. R., Park, J. C., Hag Yeon Cho, H. Y. \& Kim, S. (2018). Dental students' learning attitudes and perceptions of YouTube as a lecture video hosting platform in a flipped classroom in Korea. Journal of Educational Evaluation for Health Professions, 15(24), 1-6.

Shah, K. P., Goyal, S., Ramachandran, V., Kohn, J. R., Go, J. A., Wiley, Z., Moturu, A. et al. (2020). Efficacy of quality improvement and patient safety workshops for students: a pilot study. BMC Medical Education, 20(126), 01-10.

Sohn, S., Lee, Y. M., Jung, J., Eun Shil Cha, E. S. \& Chun, C. B. (2019). The flipped classroom model for an undergraduate epidemiology course. Korean Journal of Medical Education, 31(2), 103-113.

Tan, E., Brainard, A. \& Larkin, G. L. (2015). Acceptability of the flipped classroom approach for in-house teaching in emergency medicine. Emergency Medicine Australasia, 27(5), 453-459.

Tang, F., Chen, C., Zhu, Y., Zuo, C., Zhong, Y., Wang, N., Zhou, L., Zou, Y., \& Liang, D. (2017). Comparison between flipped classroom and lecture-based classroom in ophthalmology clerkship. Medical Education Online, 22(1), 1-9.

Wu, J. C., Chi, S. C., Wu, C.C. \& Kang, Y.-N. (2018). Helps from flipped classroom in learning suturing skill: The medical students' perspective. Plos One, 13(10), 1-13. 
Research, Society and Development, v. 10, n. 14, e434101422083, 2021

(CC BY 4.0) | ISSN 2525-3409 | DOI: http://dx.doi.org/10.33448/rsd-v10i14.22083

$\mathrm{Wu}$, S. et al. (2020). Existing contradictions and suggestions: flipped classroom in radiology courses of musculoskeletal disease under Chinese medical educational mode from medical imaging student perspective. BMC Medical Education, 20(75), 1-11.

Xiao, N., Thor, D., Zheng, M., Joshua Baek, J. \& Kim, G. (2018). Flipped classroom narrows the performance gap between low- and high-performing dental students in physiology. Advances in Physiology Education, 42, 586-592.

Yang, C., Yang, X., Yang, H. \& Fan, Y. (2020). Flipped classroom combined with human anatomy web-based learning system shows promising effects in anatomy education. Medicine, 99(46) 1-6.

Youhasan, P. et al. (2021). Exploring the pedagogical design features of the flipped classroom in undergraduate nursing education: a systematic review. BMC Nursing, 20: 50.

Zante, B., Hautz, W. E. \& Schefold, J.C. (2020) Physiology education for intensive care medicine residents: A 15 -minute interactive peer-led flipped classroom session. Plos One, 15(1), 1-12.

Zhang, X. M., Yu, J. Y., Yang, Y., Feng, C. P., Lyu, J. \& Xu, S. L. (2019). A flipped classroom method based on a small private online course in physiology. Advances in Physiology Education, 43, 345-349.

Zheng, B. \& Zhang, Y. (2020). Self-regulated learning: the effect on medical student learning outcomes in a flipped classroom environment. BMC Medical Education, 20(100), 1-7.

Zheng, B., Ward, A. \& Stanulis, R. (2019). Self-regulated learning in a competency-based and flipped learning environment: learning strategies across achievement levels and years. Medical Education Online, 25(1), 1-7. 